\title{
The effects of hippocampal lesions on two neotic choice tasks
}

\author{
DENIS MITCHELL, STEPHEN MAREN, and RAY HWANG \\ University of Southern California, Los Angeles, California
}

\begin{abstract}
We report two experiments in which rats with hippocampal lesions were tested in two neotic choice tasks that provided a clear distinction between novel and familiar alternatives. In the first experiment, rats with either dorsal or complete lesions were tested in an emergence task in which they were permitted to enter and explore a novel alley from a familiar nest box. Hippocampally lesioned animals did not differ from cortical lesioned controls on the latency to enter the novel alley, the duration of time spent in the alley, or the total number of rearings in the alley during the 1-h test. However, animals with complete hippocampal lesions were more active than the controls; they showed a perseverative tendency to return repeatedly to the familiar nest box. In the second experiment, rats with complete hippocampal lesions and cortical lesioned controls were tested in a two-bottle taste neophobia task with their familiar tap water and a novel saccharin solution. There were no differences between the groups in either the initial avoidance of the novel saccharin solution or the subsequent increase in saccharin preference across repeated tests. In both experiments, hippocampally lesioned animals showed the same initial neophobic avoidance followed by the same exploratory habituation as did the controls. We discuss the similarity of the hippocampal perseveration to behavior found in other exploratory tasks and conclude that the hippocampus is not essential for neotic information processing.
\end{abstract}

Novel stimuli elicit a variety of behavioral responses in rats, including orientation, exploratory approach, and neophobic avoidance (Barnett \& Cowan, 1976; Berlyne, 1960; Chitty, 1954; Corman \& Shafer, 1968; Mitchell, Scott, \& Williams, 1973; Montgomery, 1955; Welker, 1961). These responses are conveniently classified as neotic behaviors (Corey, 1978) and are most apparent in situations providing a choice between novel and familiar alternatives (Blanchard, Kelley, \& Blanchard, 1974; Hughes, 1968; Mitchell, 1976; Mitchell, Kirschbaum, \& Perry, 1975; Sheldon, 1969; Welker, 1957). Although the nature and magnitude of neotic behaviors are governed by numerous factors, such as age, sex, and strain, they are ultimately dependent on a habituation process that transforms novel stimuli into familiar stimuli (Bindra, 1959; Mitchell, Becnel, \& Blue, 1981; Mitchell, Koleszar, \& Scopatz, 1984; Mitchell, Winter, \& Moffitt, 1980; Welker, 1961).

The hippocampus has been postulated as playing an important role in mediating the habituation and neotic information processing that occurs in response to novel stimuli (Douglas, 1967; Gaffan, 1972; Gray \& McNaughton, 1983; Kimble, 1968; Krane, Sinnamon, \& Thomas, 1976;

We thank Justin Nast and Tanya Whitesel for their technical assistance. The research reported here was supported by NIH Grant AG05142 and the McKnight Foundation to Richard F. Thompson and a University of Southem California Dean's Fellowship to S.M. The authors are affiliated with the Neurosciences Program at U.S.C. S. Maren is now at the Department of Psychology, University of California, Los Angeles. Correspondence should be addressed to D. Mitchell, Department of Psychology, University of Southern California, Los Angeles, CA 90089-1061.
Maren, Patel, Thompson, \& Mitchell, 1993; Vinogradova, 1970). However, examination of the literature does not reveal a consensus on this issue. On the one hand, several investigators have suggested that the increased activity shown by hippocampal lesioned rats in an open field indicates a failure to habituate exploration (Blanchard, Blanchard, Lee, \& Fukunaga, 1977; Foreman, 1983; Kaplan, 1968; Kimble, 1963; Roberts, Dember, \& Brodwick, 1962; Satinder \& McGowan, 1985; Strong \& Jackson, 1970). On the other hand, the habituation of specific exploratory behaviors, such as rearing and sniffing, emitted in novel environments or in response to novel stimuli in familiar environments appears to proceed at a normal rate in animals with hippocampal lesions (Devenport, Hale, \& Stidham, 1988; Jarrard, 1968; Kohler, 1976; Nadel, 1968; Save, Poucet, Foreman, \& Buhot, 1992; Xavier, Stein, Francisco, \& Bueno, 1990). Moreover, while the failure of hippocampal lesioned rats to inhibit exploration of previously visited places in an open field is consistent with studies demonstrating $\mathrm{T}$-maze perseveration in animals with hippocampal lesions (Dalland, 1970, 1976; Leaton, 1965; Stevens \& Cowey, 1973), it is not consistent with either the prevailing view that hippocampal lesioned animals are nonexploratory (O'Keefe \& Nadel, 1978) or with conflicting data showing that such animals frequently respond randomly in a T-maze (Douglas \& Isaacson, 1964; Means, Leander, \& Isaacson, 1971; Roberts et al., 1962).

Although a number of procedural factors such as handling, prior experience with the apparatus, and test duration could conceivably account for the discrepant reports on the effects of hippocampal lesions on habituation and 
neotic information processing, it is more likely that shortcomings inherent in the frequently used open-field task are responsible for the confusion (Archer, 1973; Walsh \& Cummins, 1976). Corey (1978) has persuasively argued that the absence of a choice between novel and familiar alternatives in forced-exploration tasks precludes meaningful assessment of neotic preference. For example, ambulation in an open field, which is often interpreted as exploration (neotic approach), could just as logically be interpreted as escape behavior (neotic avoidance) or spontaneous activity (Welker, 1959; Whimbey \& Dennenberg, 1967). Similarly, the hyperactivity exhibited by animals with hippocampal lesions in open-field tasks may reflect a failure to habituate to exploration, an inability to inhibit motor activity, or reduced neophobia (Isaacson, 1974).

In the present experiments, we attempted to discriminate among these possibilities by providing rats with hippocampal lesions a clear choice between novel and familiar alternatives. In the first experiment we used an emergence task; in the second, a taste neophobia task.

\section{EXPERIMENT 1}

In this experiment, rats with dorsal or complete hippocampal lesions were tested in an emergence task. This was performed in a straight alley with an adjoining nest box in which the animals were housed for $24 \mathrm{~h}$ prior to a $1-\mathrm{h}$ exploration test. Choice tasks of this kind have several advantages over more traditional forced exploration tasks, such as the open field, because (1) they permit an unequivocal measure of neotic preference-exploratory animals can approach and neophobic animals can avoid the novel compartment; (2) they are less likely to be confounded by spontaneous motor activity; and (3) they provide a more logical basis for attributing frequently used investigatory behaviors, such as rearing, to exploration (Archer, 1973; Corey, 1978; Mitchell, 1976).

Occasional reports (e.g., Nadel, 1968) have noted differential effects of dorsal hippocampal lesions and of more complete lesions that included the ventral hippocampus on exploratory habituation. For this reason, we included groups of both lesion types to evaluate the relationships between the extent of hippocampal damage and the behavioral correlates of neophobia, habituation, and exploration.

\section{Method}

Subjects. Twenty-four male Long-Evans rats (Simonsen Labs) approximately 80 days old (286-353 g) were individually housed in an air-conditioned room on a 12:12-h light:dark cycle (lights on at $0600 \mathrm{~h}$ ). Each animal occupied a standard stainless steel isolation cage $(24.0 \times 17.4 \times 18.5 \mathrm{~cm})$ suspended over a stainless steel tray covered with a layer of woodchips. Food (Purina Lab Chow) was continuously available from stainless steel hoppers attached to the back of each cage. Tap water was continuously available from glass bottles with stainless steel spouts attached to the front of each cage.

Surgery. The animals were randomly divided into three groups; animals receiving dorsal hippocampal lesions (DHC; $n=8$ ), those receiving total hippocampal lesions (THC; $n=8$ ), and cortical controls (CORT; $n=8$ ). Before surgery, the rats were anesthetized with an intraperitoneal (i.p.) injection of sodium pentobarbital
$(65 \mathrm{mg} / \mathrm{kg})$ and mounted in a Kopf stereotaxic frame; the head position was adjusted to place bregma and lambda in the same horizontal plane. After retraction of the scalp, large ovoid holes (anteriorposterior boundaries $=-1.0$ and $-6.0 \mathrm{~mm}$ relative to bregma) were drilled bilaterally in the skull to expose the cortex overlying the hippocampus. Under visual guidance, either the overlying cortex alone or the cortex in combination with the dorsal hippocampus was aspirated. In animals designated to receive total hippocampal lesions, an attempt was made to aspirate the entire dorsal-ventral extent of the hippocampus. Following aspiration, the resultant cavity was loosely packed with Gelfoam soaked in a solution of thrombin.

Emergence Apparatus and Procedure. The emergence apparatus consisted of a set of four parallel wooden alleys $(90.5 \times 20.0 \times$ $28.5 \mathrm{~cm})$ painted gray and covered with hardware cloth $(5.1 \times 2.5 \mathrm{~cm}$ mesh). Four identical nest boxes $(24.0 \times 20.0 \times 28.5 \mathrm{~cm})$ with hinged hardware cloth tops $(0.5-\mathrm{cm}$ mesh) were separated from the alleys by guillotine doors forming the front wall of each box. The nest boxes and doors were painted black. Each box was ventilated through a 4-cm-diam grated hole centered $9 \mathrm{~cm}$ below the top of each back wall. The ventilation holes accessed a common manifold connected by flex tubing to a squirrel cage exhaust fan mounted outside the enclosure. A $15-\mathrm{cm}$-wide black wooden shelf mounted $4.5 \mathrm{~cm}$ above the nest boxes partially shaded the back portion of each box and supported a digital clock and animal identification cards. Food (Purina Lab Chow) and woodchip bedding was supplied on the floor of each nest box. Water was available from an externally mounted glass bottle with a stainless steel spout that projected through the back wall of the nest box $6.0 \mathrm{~cm}$ above the floor. The apparatus was positioned on the floor of a small enclosure $(135 \times 104 \times 155 \mathrm{~cm})$ with a rectangular opening $(90.5 \times 80.5 \mathrm{~cm})$ in the center of the top and a sliding door along one side. Indirect lighting was provided by four fluorescent tubes $(20 \mathrm{~W})$ mounted vertically on the walls of the enclosure, two behind and two in front of the apparatus. A monitor and video cassette recorder were positioned on a cart outside the enclosure behind the apparatus, and a video camera, mounted on scaffolding $260 \mathrm{~cm}$ above the floor, permitted a clear view of the entire apparatus.

Following surgery, the animals were individually housed for 10-15 days before the beginning of the experiment, when they were run in the emergence task in five consecutive sets of 4 rats each. Twenty-four hours before each test $(1800 \mathrm{~h})$, the appropriate animals were placed in the nest boxes adjacent to the alleys. Food and water were freely available in the nest boxes, and the lighting cycle was the same as that in the vivarium where the animals were housed. The test was conducted at the beginning of the dark cycle the following day. At the beginning of each test, the enclosure lights were reset to remain on during the test, the camera was turned on, and the guillotine doors were removed. The animals were permitted to enter and explore the novel alleys for $1 \mathrm{~h}$. Following the test, the animals were returned to their home cages. After each set of animals had been tested, the soiled litter and uneaten food were discarded and the entire apparatus was thoroughly cleaned and washed with a mild detergent solution. The water bottles were cleaned and refilled, and each nest box was resupplied with fresh food. Fresh woodchip bedding was supplied throughout the apparatus. Subsequent sets of animals were housed in the nest boxes on the following day, and the procedure was repeated until all five sets had been run.

Histology. To ascertain the extent of the brain lesions, the rats were sacrificed with an overdose of sodium pentobarbital and perfused across the heart with $0.9 \%$ saline followed by $10 \%$ formalin. The brains were frozen, and coronal sections ( $50 \mu \mathrm{m}$ thick) were cut at $-15^{\circ} \mathrm{C}$ on a cryostat. The sections were thaw mounted on chrome-alum/gelatin coated slides and dried. After drying, the sections were stained with cresyl violet.

Data analysis. The videotapes were scored by an investigator blind to the treatment conditions. Values were obtained for the latency to enter the novel alley (emergence latency), the number of 
entries into the novel alley during the 1-h test (emergence entries; the entire body of the rat minus the tail had to have entered the novel alley in order for an emergence entry to have been scored), the duration of time spent in the novel alley during the 1-h test (emergence duration), and the number of rearings made during the 1-h test (rearings; scored only if the rats raised both paws off the apparatus floor; consecutive rearings were scored only if the rats placed both paws back on the floor before the next rearing). From these values, the duration of time spent in the alley per emergence entry and the number of rearings made in the novel alley per emergence entry were calculated. A habituation curve for emergence duration was constructed by dividing each animal's emergence entries into three entry blocks; tapes were then rescored for each animal across each entry block. An analysis of variance (ANOVA) was performed, with the between-group factor of group (three levels: CORT, DHC, THC) and within-group factors of either 10-min block (block, six levels) or entry block (entry block, three levels). Post hoc comparisons were performed with the use of Fisher tests following significant omnibus $F$ tests. All data are presented as means \pm the standard errors of the means (SEM).

\section{Results}

Histology. Schematic diagrams showing the extent of typical lesions from animals in each group are shown in Figure 1. In general, the animals in the DHC group had lesions restricted to the dorsal aspect of the hippocampus, whereas the hippocampal damage in the THC animals included both the dorsal hippocampus and substantial portions of the ventral hippocampus. The ventral damage in the THC group tended to be concentrated in the most caudal portion of the hippocampus and, in 2 animals, intruded upon entorhinal cortex. None of the THC animals, however, had total ventral hippocampal lesions; the extreme ventrolateral portions of the hippocampus were intact, at least unilaterally, in the majority of subjects. Nonetheless, we estimate that greater than $85 \%$ of the hippocampus was aspirated in these animals, and approximately $60 \%$ in the DHC animals. With regard to extrahippocampal damage, the animals in each group possessed substantial cortical damage, including lesions in the cingulate, anterior and posterior parietal, and in some cases occipital, cortex. Quantification revealed that animals in the THC group possessed significantly more cortical damage than animals in the DHC and CORT groups (data not shown). In addition, the corpus callosum was damaged in all of the subjects. There was no subcortical damage in any of the CORT animals, although 2 animals in the DHC group had minor damage to the septal nuclei, and 1 in the THC group suffered unilateral damage to the caudate nucleus.

Behavior. Despite the tendency for THC animals to have shorter emergence latencies, the hippocampal lesioned animals did not significantly differ from the cortical lesioned controls on either the latency to enter the novel alley from the familiar nest box [CORT, $8.9 \pm 4.1 \mathrm{~min}$; DHC, $9.0 \pm 2.9 \mathrm{~min} ; \mathrm{THC}, 2.6 \pm 1.5 \mathrm{~min} ; F(2,21)=1.5, p=$ $.25]$ or the total time spent in the novel alley during the
CORT

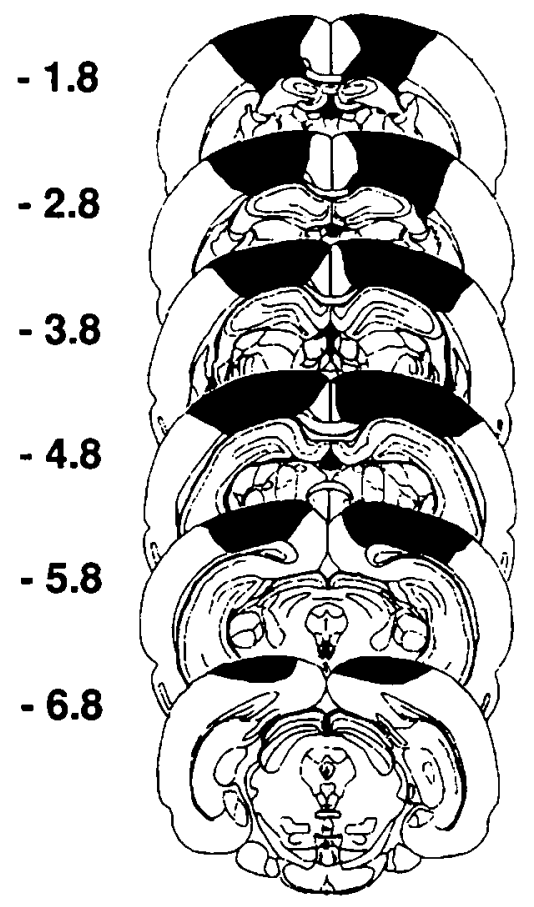

DHC

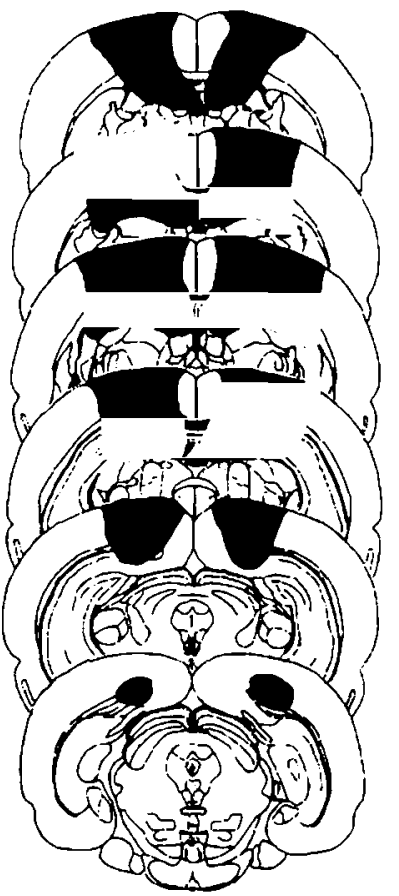

\section{THC}

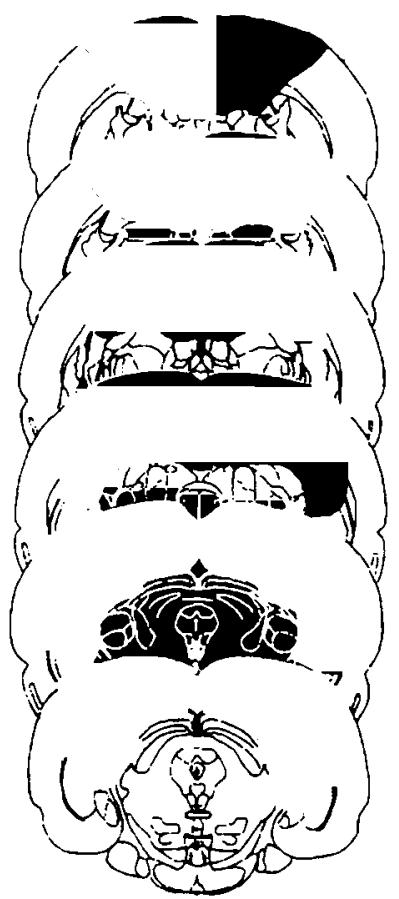

Figure 1. Schematic representation of typical lesions in animals from each of the three groups. Numeric values indicate millimeters posterior to bregma. CORT, cortical lesions; DHC, dorsal hippocampal lesions; THC, total hippocampal lesions. 
1-h test [CORT, 28.4 $\pm 6.4 \mathrm{~min} ; \mathrm{DHC}, 18.6 \pm 3.9 \mathrm{~min}$; THC, $18.4 \pm 4.7 \mathrm{~min} ; F(2,21)=1.3, p=.30]$. In contrast, hippocampal lesions significantly increased the number of emergence entries made by the animals [Figure 2A; $F(2,21)=20.1, p<.01]$. Post hoc comparisons $(p<$ .05 ) revealed that animals with total, but not dorsal, hippocampal lesions had a significantly higher number of alley entries than did the cortical lesioned controls. Moreover, post hoc comparisons performed on the significant interaction of group and block $[F(10,105)=2.6, p<.01]$ indicated that the elevated entries in the THC group occurred in the first $20 \mathrm{~min}$ of the 1-h test. Over the 1-h test, the elevated number of entries displayed by both hippocampal lesioned groups translated into a significantly re-

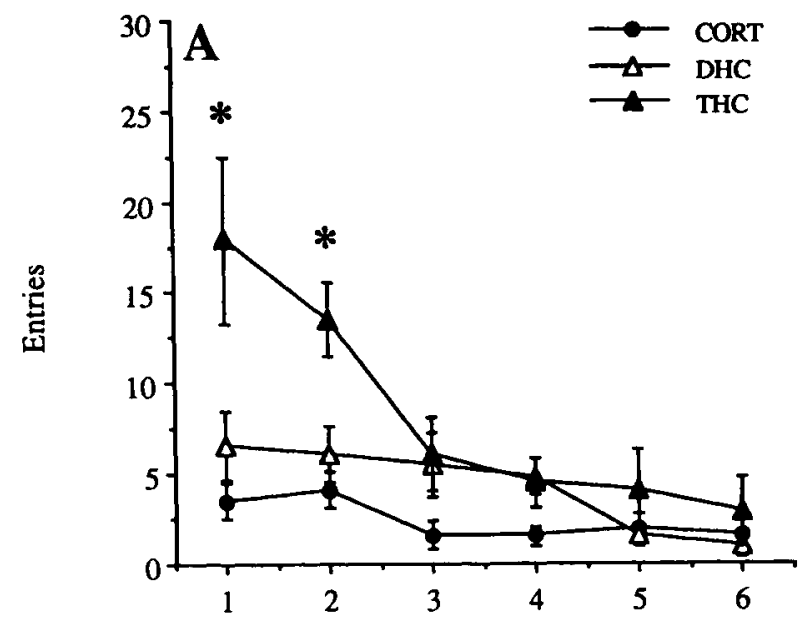

10 minute block

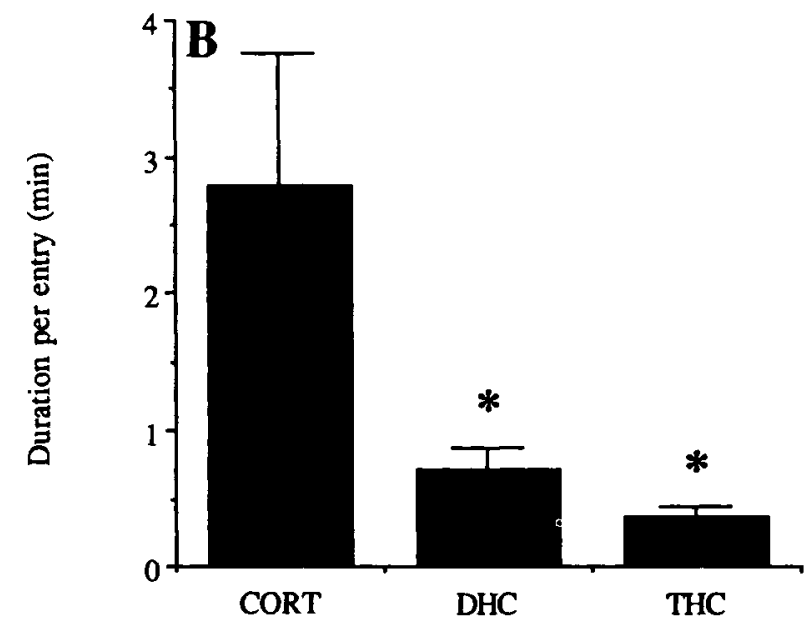

Figure 2. (A) Mean ( $\pm S E M$ ) emergence entries made during the 1-h test by each of the groups. Asterisks indicate a significant elevation $(p<.05)$ in emergence entries in the THC group compared to both the DHC and the CORT groups. (B) Mean ( \pm SEM) emergence duration per alley entry during the 1-h test. Single asterisks indicate that both hippocampal lesion groups were significantly different from cortical controls. CORT, cortical lesions; DHC, dorsal hippocampal lesions; THC, total hippocampal lesions. duced duration per alley entry for both DHC and THC animals relative to CORT animals [Figure $2 \mathrm{~B} ; F(2,21)=$ $5.3, p<.05]$. Hence, hippocampal lesioned subjects, particularly THC animals, made numerous short visits to the novel alley, whereas controls did not. Though not obvious from this analysis, the emergence behavior of animals with total hippocampal lesions was stereotyped and repetitive. The hippocampal lesioned animals tended to shuttle between the nest box and the novel alley. This behavior was extremely invariant from entry to entry; these animals generally took the same trajectory upon entering the alley, followed the same route in the alley, and explored in much the same way as they had on previous entries.

Despite this perseverative tendency, exploratory behavior (quantified as rearing activity in the novel alley) was not affected by hippocampal lesions [Figure $3 \mathrm{~A} ; \boldsymbol{F}(2,21)=$ $3.0, p=.07]$. Furthermore, rearing in the novel alley habituated across the $1-\mathrm{h}$ test session $[F(5,105)=8.0$, $p<.01]$, and the three groups did not differ in this regard $[F(10,105)=0.9, p=.53]$. However, as with the duration per entry measure, both hippocampal lesioned groups exhibited significantly fewer rearings per alley entry [Figure 3B; $F(2,21)=12.2, p<.01$ ].

Analysis of the emergence duration per entry block data revealed a main effect of entry block, indicating that all groups showed habituation over the 1 -h test [Figure 4; $F(2,42)=15.8, p<.01$ ]. A nonsignificant interaction between group and entry block $[F(2,42)=0.4, p=.79]$ revealed that all the animals habituated at the same rate. Thus, these data indicate that hippocampal lesioned animals display normal habituation, evidenced by a decrement in exploratory behavior over the 1-h test and an increased duration per entry in later entry blocks.

\section{EXPERIMENT 2}

The results of Experiment 1 indicate that hippocampal lesions disrupt neither the initial neophobia nor the subsequent habituation to a complex of novel environmental cues. Animals in the hippocampal lesioned groups did not significantly differ from cortical lesioned controls on the emergence latency, the time spent in the novel alley during the 1-h test, or the number of rearings made during the test.

In contradistinction to their performance on the latency, duration, and rearing measures, animals with total hippocampal lesions made more than three times the number of entries into the novel alley than did the cortical lesioned controls. The animals with lesions limited to the dorsal hippocampus also showed an increased, but nonsignificant, number of alley entries. The perseverative behaviors of the THC animals were similar to the exploratory behaviors of the DHC and CORT animals in that they were apparently mediated by neotic information processing. Both their exploratory behavior and their distinctive motoric patterns showed that they could distinguish between the novel alley and the familiar nest box. Moreover, they quickly became preoccupied with previously emitted (fa- 


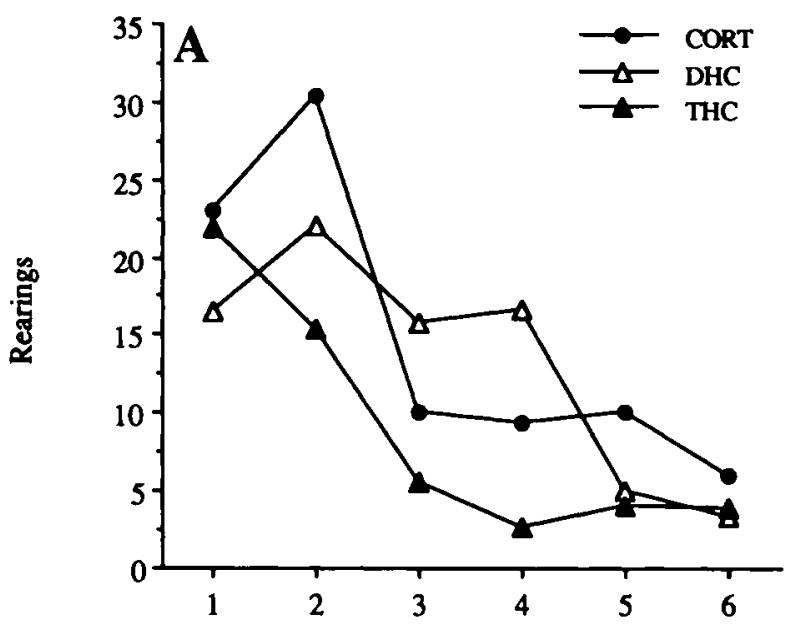

10 minute block

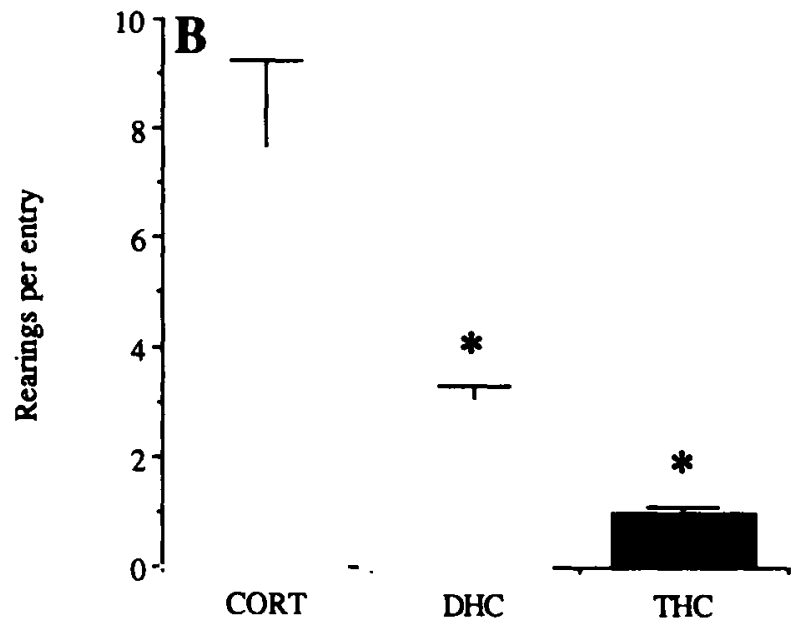

Figure 3. (A) Mean rearings made during the 1-h test by each of the groups. The groups did not differ from one another on this measure. All groups showed an early bout of exploratory activity that habituated across the test session. Error bars are not displayed because they overlap at all points. (B) Mean $( \pm S E M)$ number of rearings per alley entry made during the 1-h test. Single asterisks indicate that both hippocampal lesion groups were significantly different from cortical controls. CORT, cortical lesions; DHC, dorsal hippocampal lesions; THC, total hippocampal lesions.

miliar) responses. Though this perseverative pattern of emergence behavior is not incompatible with the conclusion that hippocampal lesions disrupt neither neophobia nor habituation, skeptical readers may need further evidence before they concur. Moreover, the finding that hippocampal lesions have no effect on neophobia is apparently inconsistent with at least one report showing attenuated taste neophobia in hippocampal lesioned animals (Krane et al., 1976), and another showing that hippocampal lesions disrupt neophobia for both taste and odor cues (Miller, Nonneman, Kelly, Neisewander, \& Isaac, 1986).

Taken together, these considerations detract from the generality of the results of Experiment 1 . We therefore decided to assess the role of the hippocampus in neotic choice behaviors further by employing a taste neophobia task. Unlike the emergence task, the taste neophobia task is free of possible motor activity confounds and does not have a prominent spatial component. Despite the differences between the tasks, however, we reasoned that the strong correlation between emergence neophobia and taste neophobia (Mitchell, 1985) and the polymodal nature of hippocampal afferent projections (Van Hoesen, Pandya, \& Butters, 1972) would predict that hippocampal lesions, which have no effect on emergence neophobia or exploratory habituation, should correspondingly have no effect on neophobia for or habituation to stimuli processed in other sensory modalities.

Accordingly, in Experiment 2, we performed extensive hippocampal lesions on the experimental animals in order to ensure that they were comparable to the THC group that had shown the most pronounced elevation in emergence entries in Experiment 1. Subsequently, both the hippocampal lesioned animals and a group of cortical lesioned controls were administered a two-bottle preference between their familiar tap water and a novel saccharin solution.

\section{Method}

Subjects and surgery. Twenty-eight male Long-Evans (Simonsen) rats approximately 70 days old (243-318 g) at the time of surgery were used as subjects. The animals were housed under conditions identical to those in Experiment 1. Food and water were

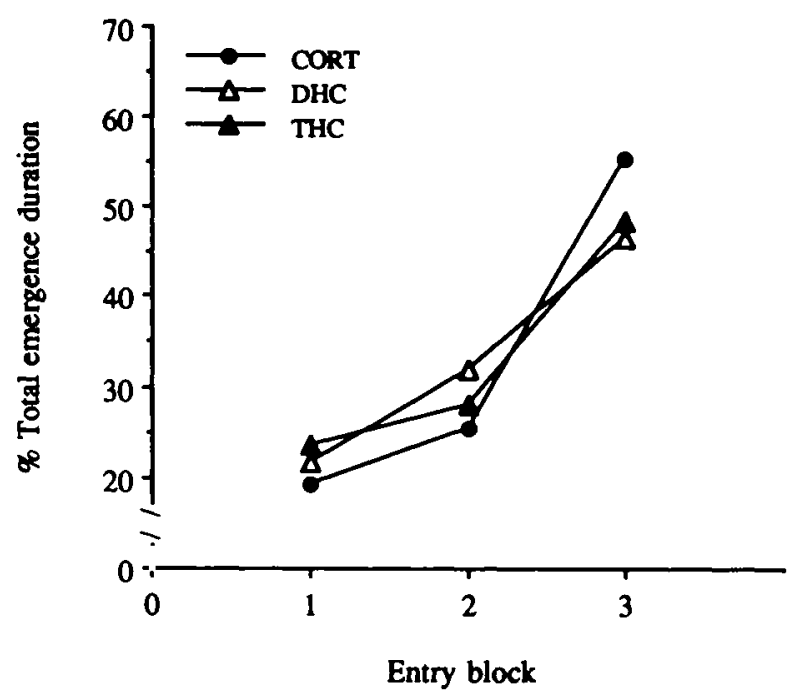

Figure 4. Mean percent of total emergence duration across three consecutive entry blocks. Entry blocks were calculated by dividing each animal's alley entries into three blocks. These entry blocks were then rescored for emergence duration and rearings. That all groups showed significant habituation is evident by a substantial increase in emergence duration across the three entry blocks. Specifically, emergence duration in the third entry block was significantly greater than that in both the first and the second entry blocks. Error bars are not displayed because they overlap at all points. CORT, cortical lesions; DHC, dorsal hippocampal lesions; THC, total hippocampal lesions. 
continuously available, except where specified in the procedure. The surgical methods were the same as in Experiment 1, except all experimental animals received total hippocampal lesions. Four animals did not recover from surgery, leaving 14 hippocampal lesioned animals and 10 cortical lesioned controls to complete the experiment. Following surgery, the animals were individually housed for 3-4 weeks before the beginning of the experiment.

Procedure. The two-phase experiment (adaptation and testing) was conducted in an air-conditioned room with a 12:12-h light:dark cycle as in Experiment 1. Three to four weeks after surgery, the animals were adapted to a restricted 30 -min water regimen. After 6 days of adaptation, all subjects were given 5 consecutive days of preference tests between water and $0.1 \%(w / v)$ sodium saccharin during the usual 30 -min fluid session. Bottle positions were counterbalanced across the manipulations. During recovery from surgery and during adaptation, the single water bottle was always placed in the center of the cage. During testing, the saccharin bottles were initially placed on the right side for half of the animals in each surgical treatment; for the other half, they were initially placed on the left side. The left-right bottle placement was alternated daily thereafter. Consumption of water and saccharin $( \pm 0.1 \mathrm{ml})$ during each 30 -min preference test was recorded for each subject. Saccharin preference was calculated by dividing saccharin consumption by total (saccharin + water) fluid consumption. Testing was done under single-blind conditions; the experimenter collecting the fluid-consumption data had no knowledge of surgical treatments. The histological procedures were identical to those in Experiment 1.

Data analysis. Saccharin preference scores from the 5-day taste neophobia test were submitted to a repeated measures ANOVA with a between-group factor of lesion (two levels: hippocampal and cortical lesions) and a within-group factor of test day (five levels). The mean total fluid consumption data were submitted to an ANOVA with one between-group factor of lesion (two levels). Planned comparisons in the form of univariate $F$ tests were carried out following significant omnibus $F$ tests in the ANOVA. All data are presented as means \pm the standard errors of the means (SEM).

\section{Results}

Histology. The histological results were similar to those reported in Experiment 1. All of the subjects in the experimental group had total hippocampal lesions and damage to the overlying cortical regions that exceeded the cortical damage in the control animals. Moreover, the corpus callosum was damaged in all of the subjects. Lesions in the controls were confined to cortical regions overlying the hippocampus, and overlapped substantially with the cortical regions destroyed in experimental animals.

Behavior. As illustrated in Figure 5, hippocampal lesions had no effect either on the initial neophobic avoidance, or on the subsequent habituation to the novel saccharin solution during the two-bottle taste neophobia test [lesion, $F(1,22)=.75, p=.40$; lesion $\times$ test day, $F(4,88)=.02, p=1.00]$. Habituation was evidenced by an increase in saccharin preference in both groups across the 5 test days [test day, $F(4,88)=82.9, p<.01$ ] Planned comparisons indicated that saccharin preference reached asymptote (approximately $80 \%$ ) on the 3rd test day, and was maintained at this level on the 4th and 5th test days. There were no group differences in the mean total fluid consumption for the 5 days of the test $[F(1,22)=$ $.09, p=.77]$.

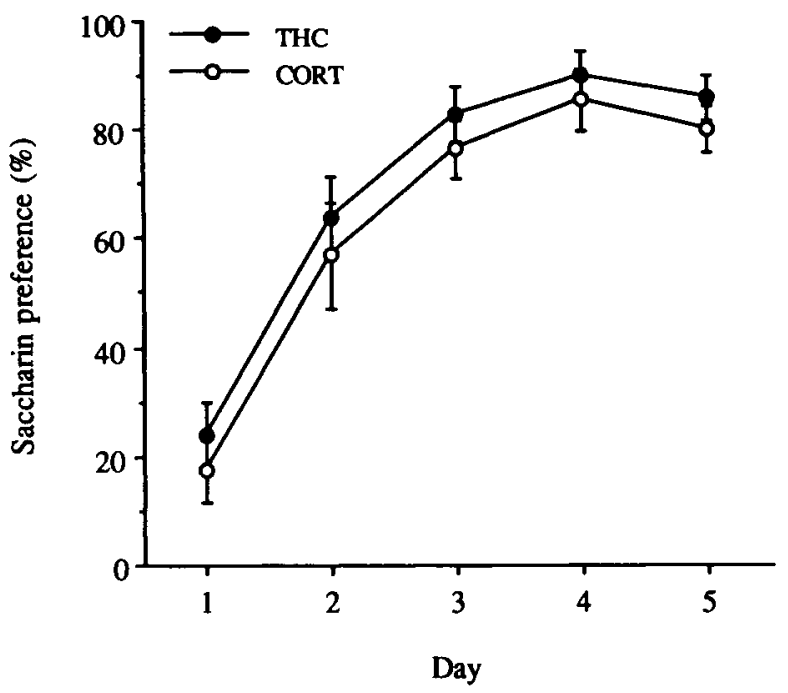

Figure 5. Mean ( $\pm S E M$ ) saccharin preference (in percent) across the 5 test days. Animals with total hippocampal lesions (THC) did not differ from cortical controls (CORT) on either their initial avoidance of the novel saccharin solution or their subsequent habituation to it.

\section{GENERAL DISCUSSION}

The present data indicate that rats with hippocampal lesions show deficits in neither habituation nor neotic information processing. In the emergence task, cortical and hippocampal lesioned animals were initially neophobic; that is, they were reluctant to enter the novel alley. This indicates that the animals had become familiar with (had habituated to) the nest box, and that habituation mediated a shift in neotic preference from neophobic avoidance to exploratory approach. Moreover, the pattern of exploratory behavior emitted by hippocampal animals in the novel alley showed a characteristic habituation curve that was similar to that exhibited by cortical controls. Likewise, when provided with a choice between a novel saccharin solution and familiar tap water, animals with total hippocampal lesions showed an initial avoidance and subsequent habituation to the novel solution that was comparable to that of the controls. Together, these data confirm and extend previous findings from several other paradigms that neophobia is not modality specific and that habituation mediates the characteristic shift in neotic preference that occurs with repeated exposure to novel stimuli (Mitchell, 1978; Mitchell et al., 1981; Mitchell et al., 1975; Mitchell et al., 1984). Our findings of intact habituation in animals with hippocampal lesions are consistent with a number of studies reporting similar data (Jarrard, 1968; Nadel, 1968; Save et al., 1992; Xavier et al., 1990).

The necessity of removing overlying cortical tissue may detract from our conclusion that the behavioral differences we observed between the groups in Experiment 1 were largely due to hippocampal and not to cortical damage. However, both the emergence latencies and the duration of time spent in the novel alley during the 1-h test by the 
cortical lesioned controls are comparable to the performance of unoperated cohorts of the same age, sex, and strain previously run under similar conditions (Mitchell, 1985). Moreover, other investigators have reported similar perseverative behaviors in hippocampal lesioned rats with minimal cortical damage (cf. Blanchard et al., 1977; Dalland, 1976; Devenport et al., 1988; Leaton, 1965).

Despite the general similarity between the hippocampal lesioned and the cortical lesioned controls in both experiments, the behavioral pattern of the hippocampal lesioned animals in the emergence task made them qualitatively different from the controls. In the method sections of both of the present experiments, we have stated that the behavioral data were collected under "blind" conditions. Yet, although the experimenter remained ignorant of the surgical treatment conditions during the collection of the taste neophobia data, this could not be said for the emergence task. This is because the hippocampal lesioned rats were not simply more active than the controls; they typically showed a characteristic sequence of stereotyped behaviors as they shuttled between the familiar nest box and the novel alley. In the typical case, animals with more extensive lesions would repeatedly emerge from the nest box, traverse the alley, rear off the floor for a moment, then rapidly return to the nest box. Moreover, for any given hippocampal lesioned animal, the details of this emergence pattern were remarkably consistent across trials: If such an animal stopped in the middle of the alley on a given visit, it almost always stopped at the same place on preceding or subsequent visits. If it reared at one spot on any one visit, it was quite likely to rear at that same spot on the majority of visits. In short, the behavioral pattern of the animals with total hippocampal lesions was perseverative: It consisted of repeated stereotyped movements executed in rapid succession through an almost invariant sequence from start to finish. The Blanchards (Blanchard \& Blanchard, 1979) have cogently pointed out that the behavior of hippocampal lesioned rats is so characteristic that the experienced observer cannot conceivably run "blind." Our observations confirm this assertion.

The perseveration of the THC rats in the emergence task is congruent with similar perseverative behaviors reported by many researchers employing other exploration tasks (for reviews, see Douglas, 1967; Gray \& McNaughton, 1983; O'Keefe \& Nadel, 1978). For example, in contrasting the activity of hippocampal lesioned animals in a novel environment with the exploratory behavior of intact animals, O'Keefe and Nadel point out that the former typically adopt a stereotyped pattern of behavior that involves increased movement and "a noticeable tendency to go back to the same areas over and over again." Likewise, the perseverative tendencies of hippocampal lesioned rats in a variety of maze learning tasks is well documented. Douglas (1967) has pointed out that experimenters frequently describe such animals as "lacking flexibility," "repeatedly reentering blind alleys," and "per- severative in their maze decisions." The behavior of the animals with total hippocampal lesions in the emergence task reported in Experiment 1 is compatible with these descriptions.

The present results are also compatible with those of Devenport et al. (1988), who reported that in both a radial maze and operant chambers, when reward was not behaviorally contingent, rats with hippocampal lesions showed a progressive decrease in behavioral variability (an increase in perseveration) across trials. However, in contrast to their view that associative hedonic information processing mechanisms such as reward expectancy mediate the perseverative behaviors of hippocampal lesioned rats (Devenport et al., 1988), our data indicate that hippocampal perseveration is mediated by nonassociative neotic information processing mechanisms such as habituation and recognition memory.

The stereotyped perseverative behaviors exhibited by the THC animals apparently interfered with their exploration of the novel alley only in the sense that it made them less efficient; they had to expend more energy (make more entries) into the alley in order to achieve the same degree of habituation as that attained by the controls. Because the animals volunteered to enter the novel alley, we are confident that any investigatory behavior, such as rearing, that occurred in the novel portion of the apparatus reflected exploration and not the occurrence of spontaneous or random activity (Corey, 1978; Mitchell, 1976). Moreover, the exploratory behaviors emitted by hippocampal lesioned animals habituated during the test session. This would not be expected if the behavior was spontaneous or random. Hence, the present data do not support the widely held view that hippocampal lesioned animals are nonexploratory (O'Keefe \& Nadel, 1978). Rather, these data suggest that the stereotyped perseveration of such animals represents a characteristic and consistent pattern of behavior in novel environments composed of increased activity that rapidly becomes fixated on neotic stimuli and responses. Apparently, the perseverative behaviors of hippocampal lesioned animals are mediated by the same neotic mechanisms of neophobia and habituation as is the exploration of intact animals.

In a similar way, the present data do not support the view that the hyperactivity of hippocampal lesioned animals in an open field is due to a habituation deficit (Foreman, 1983; Gray \& McNaughton, 1983; Roberts et al., 1962; Satinder \& McGowan, 1985; Strong \& Jackson, 1970). In the first experiment, hippocampal lesions, particularly total hippocampal lesions, produced a behavioral hyperactivity, manifested as a substantial elevation of alley entries in the emergence task. Nevertheless, the hippocampal lesioned animals showed habituation of both emergence duration and exploration during the test session. In the second experiment, in which a taste neophobia test was conducted in the animals' familiar home cages, no hyperactivity was apparent and the hippocampal lesioned animals 
showed the same habituation curves as the controls did. It therefore seems unlikely that the hyperactivity of hippocampal lesioned animals in novel environments is caused by a habituation deficit. As pointed out in the introduction, interpreting behavioral data from forced exploration tasks is equivocal. In cases in which hippocampal lesioned animals are permitted a choice between novel and familiar stimuli, habituation and exploratory behavior are usually found to be intact (Save et al., 1992; Xavier et al., 1990).

Few researchers have explicitly investigated neophobia in hippocampal lesioned animals, and those who have report disparate results in taste neophobia paradigms (cf. Krane et al., 1976; Miller et al., 1986; Nachman \& Ashe, 1974). Both Miller et al. and Krane et al. reported taste neophobia deficits in hippocampal lesioned rats, whereas Nachman and Ashe found no difference between such animals and sham-operated controls on this measure. Similarly, we found no difference between hippocampal lesioned rats and cortical lesioned controls on either the initial avoidance of the novel saccharin solution or the subsequent habituation to it. The reasons for these discrepancies are unclear. Apparently, all of these studies were run with animals with nearly total hippocampal lesions. The disruption of taste neophobia reported by Miller et al. and Krane et al. may have been due to the use of a relatively insensitive one-bottle test (calibrated drinking tubes) in the first case, and the use of novel drinking cages in both cases. Moreover, the animals in Krane et al.'s study had experience of a novel solution (sucrose) before being tested for taste neophobia with two additional novel solutions (saline and Tang). Ample data indicate that prior experience with novelty profoundly affects neotic choice behaviors (Berlyne, 1960; Corey, 1978; Mitchell, 1978; Welker, 1961).

The contrasting conditions of the two experiments reported here provide information beyond the finding that hippocampal lesioned animals are able to habituate and explore. It is probably significant that perseverative behaviors occurred only in the emergence task, a task in which motor activity can be expressed in a novel environment. In contrast, neither hyperactivity nor perseveration was elicited in the taste neophobia test conducted in the animals' familiar home cages. Hence, environmental novelty coupled with the opportunity to ambulate seems particularly potent in eliciting perseveration in hippocampal lesioned animals. This is consistent with data indicating that increased activity and perseveration in such animals is only elicited in situations presenting considerable environmental novelty (Jarrard, 1968; Kaplan, 1968).

Among the many theories proposed to account for hippocampal function, inhibitory theories seem most congruent with the present results. In the 1960s, Douglas (1967) compiled an exhaustive review of the literature and concluded that "the behavioral changes found after hippocampal lesions are almost invariably of a perseverative nature when a prepotent response is involved, implying that the basic function of the hippocampus is inhibitory."
Similarly, the Blanchards (Blanchard et al., 1977) have referred to the inability of hippocampal lesioned animals to maintain "active arrest" postures such as crouching and freezing. The present data seem to indicate that the presence or absence of neotic contextual or environmental cues is an important condition for eliciting hyperactivity and perseverative behavior in hippocampal lesioned animals.

In many ways, the behavior shown by hippocampal lesioned animals in the emergence task is reminiscent of the "blindsight" phenomenon in humans. In this instance, individuals who report blindness can, in fact, process and detect visual stimuli, although they are seemingly unaware of their ability to do so (Weiskrantz, Warrington, Sanders, \& Marshall, 1974). Likewise, hippocampal lesioned animals appear to process neotic information and habituate accordingly, yet their propensity to repeatedly visit already explored places seems to indicate that they have no recollection of their previous visits. This phenomenon is similar, in some respects, to the deficits presented by human temporal lobe amnesics who clearly benefit from experience in a number of situations, yet report no memory for those experiences (Milner, Corkin, \& Teuber, 1968; Weiskrantz, 1978). These considerations suggest that the perseveration produced by hippocampal lesions in rats may be due, in part, to a selective disruption of declarative memory (Squire \& Cohen, 1984).

In conclusion, the results of the present experiments indicate that the neotic information processing mechanisms which mediate exploratory approach, neophobic avoidance, habituation, and recognition memory are not disrupted by hippocampal lesions. If one assumes that neotic mechanisms are intact in hippocampal lesioned rats, their perseverative behaviors in exploration tasks can fruitfully be viewed as a unique expression of processes similar to those occurring in the controls. In this view, it is not so much that hippocampal lesions interfere with neotic information processing, but that they engender a preoccupation with neotic stimuli and responses that makes hippocampal lesioned animals behaviorally different from intact animals; it makes them perseverate. We suggest that this behavioral pattern of perseveration in rats with hippocampal lesions can be used as an animal model of the human temporal lobe amnesic syndrome.

\section{REFERENCES}

ARCHER, J. (1973). Tests for emotionality in rats and mice: A review. Animal Behaviour, 21, 205-235.

Barnett, S. A., \& Cowan, P. E. (1976). Activity, exploration, curiosity, and fear: An ethological study. Interdisciplinary Science Reviews, 1, 43-62.

Berlyne, D. E. (1960). Conflict arousal and curiosity. New York: McGraw-Hill.

BINDRA, D. (1959). Stimulus change, reactions to novelty, and response decrement. Psychological Review, 66, 96-103.

BlanCHARD, D. C., \& BlanChARD, R. J. (1979). Behavioral analysis of the hippocampal syndrome. Behavioral \& Brain Sciences, 2, 487-533.

Blanchard, D. C., Blanchard, R. J., Lee, E. M., \& Fukunaga, 
K. K. (1977). Movement arrest and the hippocampus. Physiological Psychology, 5, 331-335.

Blanchard, R. J., Kelley, M. J., Blanchard, D. C. (1974). Defensive reactions and exploratory behavior in rats. Journal of Comparative \& Physiological Psychology, 87, 1129-1133.

ChIrty, D. (Ed.) (1954). The control of rats and mice (Vols. 1-2). Oxford: Oxford University Press, Clarendon Press.

COREY, D. T. (1978). The determinants of exploration and neophobia. Neuroscience \& Biobehavioral Reviews, 2, 235-253.

Corman, C. D., \& Shafer, J. N. (1968). Open-field activity and exploratory behavior. Psychonomic Science, 13, 55-56.

DALLAND, T. (1970). Response and stimulus perseveration in rats with septal and dorsal hippocampal lesions. Joumal of Comparative \& Physiological Psychology, 71, 114-118.

DALLAND, T. (1976). Response perseveration of rats with dorsal hippocampal lesions. Behavioral Biology, 17, 473-484

Devenport, L. D., Hale, R. L., \& Stidham, J. A. (1988). Sampling behavior in the radial maze and operant chamber: Role of the hippocampus and prefrontal area. Behavioral Neuroscience, 102, 489-498.

Douglas, R. J. (1967). The hippocampus and behavior. Psychological Bulletin, 67, 416-442.

Douglas, R. J., IsaAcson, R. L. (1964). Hippocampal lesions and activity. Psychonomic Science, 1, 187-188.

Foreman, N. P. (1983). Head-dipping in rats with superior collicular, medial frontal cortical and hippocampal lesions. Physiology \& Behavior, 30, 711-717.

GAFFAN, D. (1972). Loss of recognition memory in rats with lesions of the fornix. Neuropsychologia, 10, 327-341.

Gray, J. A., McNaughton, N. (1983). Comparison between the behavioral effects of septal and hippocampal lesions: A review. Neuroscience \& Biobehavioral Reviews, 7, 119-188.

Hughes, R. N. (1968). Behavior of male and female rats with freechoice of two environments differing in novelty. Animal Behaviour, 16, 92-96.

IsaAcson, R. L. (1974). The limbic system. New York: Plenum.

JARRARD, L. E. (1968). Behavior of hippocampal lesioned rats in home cage and novel situations. Physiology \& Behavior, 3, 65-70.

KAPLAN, J (1968). Approach and inhibitory reactions in rats after bilateral hippocampal damage. Journal of Comparative \& Physiological Psychology, 65, 274-281.

Kimble. D. P. (1963). The effects of bilateral hippocampal lesions in rats. Journal of Comparative \& Physiological Psychology, 56, 273-283

Kimble, D. P. (1968). Hippocampus and internal inhibition. Psychological Bulletin, 70, 285-295.

KoHLER, C. (1976). Habituation after dorsal hippocampal lesions: A test dependent phenomenon. Behavioral Biology, 18, 89-110.

Krane, R. V., Sinnamon, M. H., Thomas, G. J. (1976). Conditioned taste aversions and neophobia in rats with hippocampal lesions. Journal of Comparative \& Physiological Psychology, 90, 680-693.

LEATON, R. N. (1965). Exploratory behavior in rats with hippocampal lesions. Journal of Comparative \& Physiological Psychology, 59, 325-330.

Maren, S., PAtel, K., Thompson, R. F., \& Mitchell, D. (1993). Individual differences in emergence neophobia predict magnitude of perforant-path long-term potentiation (LTP) and plasma corticosterone levels in rats. Psychobiology, 21, 2-10.

Means, L. W., Leander, J. D., \& IsAacson, R. L. (1971). The effect of hippocampectomy on alternation behavior and response to novelty. Physiology \& Behavior, 6, 17-22.

Miller, J. S., Nonneman, A. J., Kelly, K. S., Neisewander, J. L., \& ISAAC, W. L. (1986). Disruption of neophobia, conditioned odor aversion, and conditioned taste aversion in rats with hippocampal lesions. Behavioral \& Neural Biology, 45, 240-253.

Milner, B., Corkin, S., \& Teuber, H. L. (1968). Further analysis of the hippocampal amnesic syndrome: 14-year followup study of H.M. Neuropsychologia, 6, 215-234.

MitCHELL, D. (1976). Experiments on neophobia in wild and labora- tory rats: A reevaluation. Journal of Comparative \& Physiological Psychology, 90, 190-197.

MitChfit., D. (1978). The psychological rat vs. the ethological rat Two views of the poison avoidance behavior of the rat compared. Animal Learning \& Behavior, 6, 121-124.

MitCHELL, D. (1985, November). Emergence neophabia predicts conditioned taste aversion magnitude. Paper presented at the annual meeting of the Psychonomic Society, Boston, MA.

Mitchell, D., Becnel, J. R., \& Blue, T. (1981). The neophobiaoptimality explanation of contrafreeloading rats: A reassessment. Behavioral \& Neural Biology, 32, 454-462.

Mitchell, D., Kirschbaum, E. H., Perry, R. L. (1975). Effects of neophobia and habituation on the poison-induced avoidance of exteroceptive stimuli in the rat. Journal of Experimental Psychology: Animal Behavioral Processes, 104, 47-55.

Mitchell, D., Koleszar, A. S., SCopatz, R. A. (1984). Arousal and T-maze choice behavior in mice: A convergent paradigm for neophobia constructs and optimal arousal theory. Leaming \& Morivation, 15, 287-301.

Mitchell, D., Scott, D. W., Williams, K. D. (1973). Container neophobia and the rat's preference for earned food. Behavioral Biology, 9. 613-624.

Mitchell, D., Winter, W., Moffit, T. (1980). Cross-modality contrast: Exteroceptive context habituation enhances taste neophobia and conditioned taste aversions. Animal Learning \& Behavior, 8 , 524-528.

Montgomery, K. C. (1955). The relation between fear induced by novel stimulation and exploratory behavior. Joumal of Compararive \& Physiological Psychology, 48, 254-260.

NACHMAN, M., ASHE, J. H. (1974). Effects of basolateral amygdala lesions on neophobia, learned taste aversions, and sodium appetite in rats. Joumal of Comparative \& Physiological Psychology, 87, 622-643.

NADEL, L. (1968). Dorsal and ventral hippocampal lesions and behavior Physiology \& Behavior, 3, 891-900.

O'KeEFE, J., NADEL, L. (1978). The hippocampus as a cognitive map Oxford: Oxford University Press, Clarendon Press.

Roberts, W. W., Dember, W. N., Brodwick, M. (1962). Alternation and exploration in rats with hippocampal lesions. Joumal of $\mathrm{Com}$ parative \& Physiological Psychology, 55, 695-700.

Satinder, K. P., \& MCGowan, R. A. (1985). Hippocampal lesions and open-field defecation in rats. Psychological Reports, 56, 627-630.

Save, E., Poucet, B., Foreman, N., \&uhot, M.-C. (1992). Ob.ject exploration and reactions to spatial and nonspatial changes in hooded rats following damage to parietal cortex or hippocampal formation. Behavioral Neuroscience, 106, 447-456.

Sheldon, A. B. (1969). Preference for familiar versus novel stimuli as a function of the familiarity of the environment. Journal of Comparative \& Physiological Psychology, 67, 516-521.

SQUiRE, L. R., \& COHEN, N. J. (1984). Human memory and amnesia In G. Lynch, J. L. McGaugh, \& N. M. Weinberger (Eds.), Neurobiology of learning and memory (pp. 3-64). New York: Guilford.

Stevens, R., \& Cowey, A. (1973). Effects of dorsal and ventral hippocampal lesions on spontaneous alternation, learned alternation and probability learning in rats. Brain Research, 52, 203-224.

Strong, P. N., \& JACksON, W. J. (1970). Effects of hippocampal lesions in rats on three measures of activity. Joumal of Comparative \& Physiological Psychology, 70, 60-65.

Van Hoesen, G. W., Pandya, D. N., Butters, N. (1972). Cortical afferents to the entorhinal cortex of the rhesus monkey. Science, 175 , 1471-1473.

VinogradovA, O. S. (1970). Registration of information and the limbic system. In G. Horn \& R. A. Hinde (Eds.), Short-term changes in neuronal activity and behaviour (pp. 95-140). Cambridge: Cambridge University Press.

WALSH, R. N., Cummins, R. A. (1976). The open-field test: A critical review. Psychological Bulletin, 83, 482-504.

WeISKRANTZ, L. (1978). A comparison of hippocampal pathology in 
man and other animals. In K. Elliot \& J. Whelan (Eds.), Functions of the septo-hippocampal system (pp. 373-387). Amsterdam: Elsevier.

Weiskrantz, L., Warrington, E. K., Sanders, M. D., \& MarSHALL, J. (1974). Visual capacity in the hemianopic field following a restricted occipital ablation. Brain, 97, 709-728.

WELKER, W. J. (1957). "Free" versus "forced" exploration of a novel situation by rats. Psychological Reports, 3, 95-108.

WELKER, W. J. (1959). Escape, exploratory, and food-seeking responses of rats in a novel situation. Journal of Comparative \& Physiological Psychology, 52, 106-111.

WELKER, W. J. (1961). An analysis of exploratory and play behavior in animals. In D. W. Fiske \& S. R. Maddi (Eds.), Functions of varied experience. Homewood, IL: Dorsey Press.
Whimbey, A. E., Dennenberg, V. H. (1967). Two independent behavioral dimensions in open-field performance. Journal of Comparative \& Physiological Psychology, 63, 500-504.

Xavier, G. F., Stein, C., Francisco, O., \& Bueno, A. (1990). Rats with dorsal hippocampal lesions do react to new stimuli but not to spatial changes of known stimuli. Behavioral \& Neural Biology, 54, 172-183.

(Manuscript received December 22, 1992; revision accepted for publication April 26, 1993.) 